\title{
Depletion of glycosaminoglycans decreases contact stiffness of single collagen I fibrils in aqueous
} solution

\author{
Jonathan Roth, Cody L. Hoop, Jonathan K. Williams, Robert Hayes, ${ }^{*}$ and Jean Baum * \\ Department of Chemistry and Chemical Biology, Rutgers, The State University of New Jersey, \\ Piscataway, NJ \\ *Corresponding Authors: Jean Baum and Robert Hayes \\ E-mail: jean.baum@rutgers.edu or rob.hayes@rutgers.edu \\ Running Title: GAG depletion decreases collagen stiffness
}

Keywords: extracellular matrix, collagen, fibril, glycosaminoglycans, atomic force microscopy, solution AFM, force mapping, indentation, hydration 


\begin{abstract}
Collagen fibrils serve as the molecular scaffolding for connective tissues throughout the human body and are the most abundant protein building blocks in the extracellular matrix $\left(\mathrm{ECM}^{1}\right)$. Glycosaminoglycans (GAGs) are an important class of polysaccharides in the ECM that mediate collagen fibril development, cell adhesion and motility, wound healing, inter alia. Depletion or mis-regulation of GAGs has been shown to be intimately related to diseases such as cancer, hyperglycemia, and glaucoma and is often associated with changes to the mechanical properties of the ECM. However, the molecularlevel impact of GAGs on ECM mechanobiology is not understood. Here, high resolution atomic force microscopy is used to assess perturbations to nanoscale topography and mechanical properties of single collagen fibrils upon GAG depletion in aqueous solution. Atomic force microscopy fast force mapping shows that GAG depletion reduces the contact stiffness of individual fibrils, lowering the indentation modulus by half compared to native fibrils, while retaining D-banding, a key structural motif. These results shed new light on the functional role of GAGs and may aid in strategies to treat diseases that impair ECM mechanobiology.
\end{abstract}

\section{Introduction}

Fibrillar collagen is a fundamental structural and functional protein and a major component of the extracellular matrix (ECM) in human tissues (1). Its interactions with molecular partners are significant for cellular function (24) and many are sensitive to the mechanics of their environment. Misregulation of ECM mechanobiology leads to a number of diseases, including cancer and fibrosis $(3,5,6)$. For example, tumor cell proliferation has been linked to increased stiffness of the ECM (5). Recently, much scientific interest has focused on understanding the mechanisms by which the ECM orchestrates the mechanics of constituent collagen fibrils (7). However, this is complicated in part by the hierarchy of length scales in collagen self-assembly (Figure 1a) from triple helical monomers to fibrils to macro-scale tissues (which may in turn exhibit different stress-strain relationships). Adding to the complexity, the ECM mechanobiology is defined by multiple physical properties, such as bending, indentation, and tensile moduli, each of which are influenced by the multiple hierarchical levels of the collagen assembly $(1,8,9)$. Experimentally elucidating these mechanical properties at any length scale of the ECM under physiological conditions remains a challenge. Probing the mechanics of individual collagen fibrils, fundamental building blocks of the ECM, in an aqueous environment will provide valuable insight into the role of this hierarchy in ECM mechanobiology.

The highly conserved collagen assembly is regulated by proteoglycans (PGs), in which protein cores are bound to anionic polysaccharide chains, called glycosaminoglycans (GAGs) (10,11). A large diversity of PGs and GAGs are distributed across connective tissues for proper ECM activity (10). In all tissues, PGs recognize a number of specific amino acid sequence motifs on collagen fibrils (12-15) to act as part of the ECM scaffolding (Figure 1a). However, their specific structural roles have been debated. They have been proposed to form fibril-fibril crosslinks within higher order fibril bundles, or fibers, to in turn regulate load bearing (16). In contrast, the hydrophilic GAG chains may not couple individual fibrils, but rather isolate them to enable fibril sliding under load and preserve fiber structural integrity (17). A recent study proposed that GAGs may perform both roles on collagen fibrils (18).

In addition to their structural roles, GAGs have been shown to be essential for wound healing, cell adhesion, and cell motility $(10,19,20)$. As such, depletion or misregulation of GAGs is often associated with natural aging (21) or debilitating diseases (22-24). Notably, it has been proposed that misregulation of GAGs perturbs the mechanobiology of the ECM, leading to a loss of biological function and aberrant cell activity (7). However, the mechanism and extent to which GAGs alter the mechanical properties of individual collagen fibrils has not been elucidated.

Here, we investigate the influence of GAG content on individual collagen fibrils in aqueous buffer solution via atomic force microscopy (AFM). AFM is a powerful and versatile experimental technique in biophysics. 
Recently, the emergence of sophisticated AFM-imaging modes has enabled direct, highresolution images of structure or mechanical/surface properties of biological systems in situ under physiological aqueous conditions $(25,26)$. For collagen, many AFM studies have confirmed the characteristic $\sim 67$ nm D-banding of surface adsorbed fibrils (2732). However, these studies have largely been restricted to measurements in air, which itself complicates imaging due to trace water adsorbed to the fibrils. Given that most collagenous tissue functions in a fully hydrated environment, its interaction in water with biological molecules such as GAGs is of paramount importance. In this study, two advanced AFM imaging modes are used to study the association of GAGs with collagen fibrils derived from rat tail tendons in aqueous solution. First, amplitude-modulation (AM-) AFM experiments resolve the characteristic Dbanding and hydrated nanostructure. Second, complementary fast force mapping (FFM-) AFM experiments examine how GAGs alter individual collagen fibril mechanics in nanoscale space in aqueous solution. Together, these results present a molecular-level picture of GAG-collagen fibril interactions under physiological conditions.

\section{Results and Discussion}

\section{Resolving collagen fibril D-banding in solution.}

Collagen I fibrils display a canonical repeating $67 \mathrm{~nm}$ banding pattern that is produced by the staggered arrangement of triple helical tropocollagen units, referred to as D-banding (9) (Figure 1a). AM-AFM confirms such banding is present in rat tail collagen fibrils adsorbed to a glass slide in air and when immersed in PBS buffer solution (Figure 1bc). Here, the sinusoidal periodicity in the height along the main fibril axis is observed in both imaging environments, with alternating high ("overlap," yellow), and low ("gap," red) regions. Height profiles extracted from the apex of the collagen fibrils in air reveal a distinct sinusoidal oscillation of overlap and gap regions (Figure S1a,c). Although this periodicity in the height profiles is not as clear from images in PBS, it is discernible in the phase channel (Figure S2a-b). Twodimensional Fourier transforms of the phase channel from imaging native collagen fibrils revealed an average periodicity of $65.6 \pm 1.0$ $\mathrm{nm}(\mathrm{N}=5)$ in air and $65.8 \pm 0.8 \mathrm{~nm}(\mathrm{~N}=5)$ in PBS (Table 1). Thus, the fibril periodicity, a structural hallmark of fibrillar collagens, is maintained in an aqueous environment.

\section{The fibril nanoscale structure is maintained upon depletion of GAGs.}

We then asked if this characteristic structure of individual collagen fibrils is impacted by the concentration of GAGs in their environment. We used AM-AFM to probe perturbations to the fibril topography upon treatment with chondroitinase. The chondroitinase treated fibrils observed here have a $76 \%$ reduction in GAG content compared to native counterparts as measured using a DMMB (dimethylmethylene blue) assay (33). Chondroitinase-treated collagen fibrils display D-band periodicities of $66.0 \pm 0.2 \mathrm{~nm}$ in air $(\mathrm{N}=5)$ and $66.2 \pm 1.1 \mathrm{~nm}$ in PBS $(\mathrm{N}=4)$, as measured from 2D Fourier transforms of the phase channel (Table 1). This is consistent with the native fibril data and with previously reported AFM results of chondroitinase-treated tendons in air (17).

Another assessment of fibril surface topography is the overlap-gap step height. This is the difference between the maximum height of the overlap and the minimum height of the gap (Figure S4). Upon chondroitinase treatment, the overlap-gap step height also remained unchanged, as chondroitinase-treated collagen fibrils exhibited an overlap-gap step height of $3.6 \pm 2.7 \mathrm{~nm}$ in air compared to $3.6 \pm$ $2.4 \mathrm{~nm}$ in native collagen fibrils (Figure S7a). Step-heights in liquid were unable to be determined as gap and overlap regions were indistinguishable across height profiles (Figure S1b, d).

Submersion of collagen fibrils in aqueous solution causes swelling due to increased hydration (34). Hydration was assessed by measuring the average height along the apex of the fibril surface. The swelling ratio is defined as the ratio of height in solution relative to that in air. For native fibrils, the swelling ratio is found to be $1.79 \pm 0.06(\mathrm{~N}=5)$, confirming 
swelling in solution. This suggests a radial enlargement due to entrained water, consistent with previous measurements on collagen (34) or other fibrillar units (35). GAG-depleted fibrils were found to have a swelling ratio of $1.9 \pm 0.2(\mathrm{~N}=5)$ (Figure $\mathrm{S} 7 \mathrm{~b})$. The fibrils measured in the GAG-depleted state have a notably broader distribution of swelling ratios (Figure S7b). This may be due to heterogeneity of fibrils in the GAG-depleted environment. While the GAG concentration in the bulk solution is reduced by $76 \%$, GAG content of individual fibrils may vary from fibril-to-fibril and at different positions along the fibril. Thus, those fibrils with higher swelling ratios are expected to have greater reduction in GAG content. Overall, the data show that GAG association does not significantly impact fibril periodicity, topography, and hydration. This indicates that the collagen fibril nanoscale structure is preserved upon GAG depletion in aqueous solution.

\section{AFM force mapping reveals a decreased indentation modulus of individual collagen fibrils upon GAG depletion.}

Despite the importance of ECM mechanics to cellular processes, how GAG depletion influences the mechanical properties of individual collagen fibrils is not well understood. We used FFM-AFM to determine the indentation modulus, a measure of local nanoscale contact stiffness, of individual collagen fibrils in solution in native and GAGdepleted states. Figure 2 presents a 1 x $1 \mu \mathrm{m}$ force map of native and GAG-depleted collagen fibrils, respectively. Unlike in Figure 1 , which is an amplitude-modulated height image, it is important to emphasize that these images are 2D maps displaying the indentation modulus of each pixel (Figure S6). This enables mechanical data to be assessed at different locations within the D-bands along the fibrils.

Notably, D-banding is evident in both force maps; the overlap region systematically has a larger indentation modulus than the gap regions, leading to a distinct light/dark/light patterning every $\sim 67 \mathrm{~nm}$ down the long axis. While the demarcation between gap/overlap is less well-defined than for AM-AFM data, the results show that even in solution, collagen mechanics oscillate on the nanoscale as dictated by the underlying D-banded structure. Specifically, the staggered arrangement of monomer units leads to a more elastic gap region compared to overlap regions due to the reduced molecular density. Previous studies have reported a $20 \%$ increase in modulus of the overlap relative to the gap as measured by fast nanoindentation. This increase was attributed to the $20 \%$ higher tropocollagen density in the overlap relative to the gap regions due to the staggered arrangement of monomer units, leading to a more elastic gap region (32).

Native and chondroitinase-treated collagen fibrils display important differences in the forces measured and a significant reduction in average local indentation modulus is evident. In Figure 2a, overlap regions have moduli of $\sim 9 \mathrm{MPa}(\mathrm{red})$ on the native fibril compared to $\sim 5 \mathrm{MPa}$ (light blue) for treated fibrils. Likewise, corresponding gap regions shift from $\sim 7 \mathrm{MPa}$ (yellow) to $\sim 2 \mathrm{MPa}$ (dark blue) upon treatment while preserving the native Dbanded structure. To better understand these differences we focused on the 256 force curves along the apex of each fibril (Figure 2c). The native, untreated collagen fibrils exhibit a mean indentation modulus of $6.4 \pm 1.4 \mathrm{MPa}$, using an indentation speed of $16 \mu \mathrm{m} / \mathrm{second}$ with a broad distribution of values over the fibrils measured (Figure 2a, c, S7c). This is consistent with reported indentation moduli of collagen fibrils with similar indentation speeds (32). For treated fibrils, both the average indentation modulus $(2.9 \pm 1.0 \mathrm{MPa})$ and distribution is reduced (Figure 2b, c). This indicates that GAG depletion reduces fibril stiffness on the nanoscale. We observe that the indentation modulus is reduced in both the overlap and gap regions upon GAG depletion, suggesting that GAG content is reduced across the overlap and gap regions. This is consistent with binding domains for different GAGs being identified in both overlap and gap regions (12-15,36). Thus, while GAG depletion does not significantly impact the topography of individual collagen fibrils, it does modulate the contact stiffness of individual fibrils. We expect that this perturbation at the collagen fibril building block level will propagate to higher order collagen assemblies, modulating the 
mechanobiology of the ECM and potentially disrupting numerous cellular processes that are reliant on ECM mechanics.

One possible contribution to the increased indentation modulus induced by GAGs is increased osmotic pressure. In solution, the negatively charged GAGs may attract ions (or water molecules) and create an area of highdensity liquid. This type of structural change in solution would induce an osmotic pressure on the fibril surface $(20,37,38)$, which will effectively compress the collagen fibrils. As a result, the molecular distance between adjacent monomers within the fibril will decrease, making the fibril more tightly packed and dense with monomers, leading to a stiffer fibril (i.e. larger indentation modulus). When GAGs are removed this compression will no longer occur, allowing for more water to inhabit the interfibrillar space and increasing the molecular distance of adjacent monomers, leading to a softer fibril (i.e. smaller indentation modulus). To simulate the effect of osmotic pressure on individual collagen fibrils in solution, investigators monitored fibril height and indentation modulus upon increasing concentrations of polyethylene glycol (PEG) and varying tensile strain (34). They observed reduced fibril height, interpreted as fibril dehydration, and increased indentation modulus with increased PEG concentrations (34). This suggested that the mechanical properties of collagen fibrils can be modulated by osmotic pressure and hydration. Our results that show a higher measured indentation modulus in the native collagen fibrils relative to the GAG-depleted fibrils (Figure 2) are consistent with this.

In conclusion, we have shown that under physiological solution conditions, GAG depletion decreases stiffness of individual collagen fibrils while maintaining their native nanoscale topography. It is increasingly clear that alteration of GAG concentration is intimately associated with the progression of aging and diseases, which leads to disruptions in the mechanical landscape of the ECM. We expect that mechanical properties at the fibril hierarchy will influence the mechanobiology of the ECM, and mechanical decline may compromise cell-ECM interactions and reliant cell processes. Still, further research will be required to make a direct association between GAG depletion and cell activity or how the effect propagates longer length scales beyond the fibril building block. We now have a greater understanding of the effects of GAG depletion on collagen fibril stiffness, which could aid in understanding how diseases associated with ECM mechanobiology manifest and the development of new treatment strategies. Future studies will address the impact of GAGs on collagen fibril dynamics within the ECM, which are important for functional interactions with cell receptors and biomolecules.

\section{Experimental Procedures}

Preparation of collagen fibril samples and chondroitinase treatment.

Rat tail tendons were harvested from frozenthawed rat tails. Chondroitinase treatment of tendons was performed in a similar manner to previously published methods (17). In short, sections of tendon several centimeters in length were cut from the exposed tail tendons. For chondroitinase treatment, the tail tendon sections were placed into a $4 \mathrm{~mL}$ solution of $0.15 \mathrm{U} / \mathrm{mL}$ chondroitinase $\mathrm{ABC}$ (Sigma-Aldrich) in $0.1 \mathrm{M}$ sodium acetate and $0.1 \mathrm{M}$ Tris- $\mathrm{HCl}, \mathrm{pH} 8$. For the untreated control, fibrils were placed in the same buffer in the absence of chondroitinase. These are referred to as native fibrils. The tendons were incubated in this solution at $37^{\circ} \mathrm{C}$ overnight. Afterwards, the tendon sections were rinsed with fresh PBS buffer solution ( $\mathrm{pH}$ 7.4) and smeared onto a microscope glass slide in order to deposit collagen fibrils onto the surface. Prior to experiments, microscope glass surfaces were sonicated in ultrapure water, rinsed with ethanol, and then dried with ultra-high purity nitrogen. The samples were then washed with ultrapure water and allowed to dry for one hour in a laminar flow hood (1300 Series A2, Thermo Scientific). Once dry, a stereoscope was used to locate and assess fibril deposition prior to AFM experiments.

\section{Quantification of GAG depletion.}

Sections of separately prepared rat tail tendon were weighed, and then given the same chondroitinase/control treatment provided above. 
Tendons were then rinsed with water and placed into $1 \mathrm{~mL}$ of a papain solution $(500 \mu \mathrm{g}$ papain, 0.1 $\mathrm{M}$ dibasic sodium phosphate, $0.144 \mathrm{M}$ EDTA, 0.1 $\mathrm{M}$ L-cysteine) at $60^{\circ} \mathrm{C}$ for 24 hours to digest the tendons. The resulting solution was stained with dimethylmethylene-blue and absorbance was measured at $525 \mathrm{~nm}$. A standard curve of varying chondroitin sulfate concentrations $(0.25-1.5$ $\mu \mathrm{g} / \mathrm{mL}$ ) was used to quantify the GAG concentration in the treated and control samples.

\section{Atomic force microscopy (AFM).}

Amplitude modulation (AM-) imaging and fast force mapping (FFM-) experiments were performed on a Cypher ES AFM (Asylum Research, Oxford Instruments). Silicon nitride cantilevers AC240 and Biolever-mini BL-AC40TS sourced from Oxford Instruments were used for AM imaging in air and liquid, respectively. These cantilevers have a nominal spring constant of $\mathrm{k}_{\mathrm{c}}=$ $2.0 \mathrm{Nm}^{-1}$ and $0.1 \mathrm{Nm}^{-1}$, and radii of $\mathrm{r}=7 \mathrm{~nm}$ and 8 $\mathrm{nm}$, respectively. Image and force measurements were acquired at $25^{\circ} \mathrm{C}$. The glass microscope slide surface with deposited collagen fibrils was mounted on a steel puck within the AFM box (a sealed enclosure). Samples were first imaged in air to locate and characterize the collagen fibrils. Before liquid experiments, the spring constant of the cantilever was first determined in air using the GetReal function in the Asylum Research AFM software, which is a combination of the Sader (39) and thermal noise method. The inverse optical lever sensitivity (InVOLS) was then determined by averaging the results of ten force curves on a sapphire surface in PBS buffer. Liquid experiments were completed in a droplet of approximately $0.25 \mathrm{~mL}$ of fresh $10 \mathrm{mM}$ PBS buffer, $\mathrm{pH} 7.4$ solution on the glass microscope slide surface at $25^{\circ} \mathrm{C}$. To minimize thermal drift, this setup was allowed to equilibrate for at least one hour in the AFM prior to imaging. To facilitate obtaining high quality AM images in liquid conditions, the tip was photothermally excited at its resonance frequency using blueDrive (Asylum Research, Oxford Instruments).

\section{Fast Force Mapping (FFM).}

FFM experiments were performed immediately following AM-AFM imaging on the same fibrils characterized in air and liquid. The same BL-
AC40TS AFM probe as referenced above was used for all nanoindentation experiments. Force maps were acquired at a scan size of $1 \mu \mathrm{m} \times 1 \mu \mathrm{m}$ with a pixel resolution of $256 \times 256$ points, Z-rate of $20 \mathrm{~Hz}$, indentation speed of $16 \mu \mathrm{m} / \mathrm{sec}$, and a setpoint of $2 \mathrm{nN}$. This setpoint was used specifically to ensure that the indentation did not exceed $15 \%$ of the fibrils' total height. The indentation moduli of the fibrils were then determined from the force maps produced using the analysis of the matrix of force curves as described below.

\section{Image Analysis.}

Periodicity. 2D Fourier Transforms were performed on the phase channel of the AM images of collagen fibrils in both air and liquid using the Gwyddion software (40). Profiles were then drawn on the transformed images which then revealed the periodicity of the fibrils.

Swelling Ratio and Overlap-Gap Step Height. To calculate the swelling ratio, the average height along the apex of a fibril in liquid was divided by the average height of the same fibril in air. For determination of the overlap-gap step height from the AM images, sine curves bearing the periodicity of the respective fibrils were fitted to height profiles along the apex of the corresponding fibril. The maxima/minima values of the sin curve were strictly used to determine the corresponding overlap/gap height values used along the fibrils. Adjacent overlap and gap values were then subtracted to obtain the step heights and this was performed across an entire height profile (Figure S4). Furthermore, some chondroitinase treated fibrils imaged in air showed signs of debris occurring along the height profiles, these areas were not taking into consideration for determining the average height of the fibril or step height (Figure S5). When the treated fibrils were immersed in PBS, visual inspection revealed that there was no evidence that the debris remained on the fibrils.

\section{Post-Processing Analysis.}

FFM data sets were analyzed using in-house Matlab (R2018b) scripts. In brief, indentation moduli were calculated for each force curve iteratively using the methods detailed by Andriotis 
and coworkers (41). The resulting 256x256 matrix of FFM indentation moduli were then reconstructed in Cartesian space as a scaled colormap (Figure 2a-b, main text). Box and whisker plots were derived from a subset of five FFM images for each treatment. Here, force curve data at the height apex along the fibril longitudinal axis was extracted (256 moduli), and pooled among treatments. For this study, the apex corresponds to the values along a straight line created between two of the highest values at opposite ends of the fibril. A one-way analysis of variance (ANOVA) was performed, and the moduli data from each treatment condition are shown as a box and whisker in the main text Figure 2c.

\section{Data Availability}

Data presented is available upon reasonable request to JB.

\section{Supporting Information}

This article contains supporting information. 
bioRxiv preprint doi: https://doi.org/10.1101/2020.02.07.935932; this version posted May 10, 2021. The copyright holder for this preprint (which was not certified by peer review) is the author/funder. All rights reserved. No reuse allowed without permission.

\section{Acknowledgements}

The authors acknowledge Dr. Joseph Freeman and Michael Pellegrini of Rutgers University for assistance with rat tail procedures.

\section{Funding and Additional Information}

This work was supported by NIH grants GM045302 and GM136431 to JB and an Exploratory Research Seed Grant from Rutgers University to RH. The content is solely the responsibility of the authors and does not necessarily represent the official views of the National Institutes of Health.

\section{Conflicts of interest}

The authors declare that they have no conflicts of interest with the contents of this article.

\section{References}

1. Holmes, D. F., Lu, Y., Starborg, T., and Kadler, K. E. (2018) Collagen Fibril Assembly and Function. Curr Top Dev Biol 130, 107-142

2. Jansen, K. A., Donato, D. M., Balcioglu, H. E., Schmidt, T., Danen, E. H., and Koenderink, G. H. (2015) A guide to mechanobiology: Where biology and physics meet. Biochim Biophys Acta 1853, 3043-3052

3. Lu, P., Weaver, V. M., and Werb, Z. (2012) The extracellular matrix: a dynamic niche in cancer progression. J Cell Biol 196, 395-406

4. $\quad$ Engler, A. J., Sen, S., Sweeney, H. L., and Discher, D. E. (2006) Matrix elasticity directs stem cell lineage specification. Cell 126, 677-689

5. Jaalouk, D. E., and Lammerding, J. (2009) Mechanotransduction gone awry. Nat Rev Mol Cell Biol 10, 63-73

6. $\quad$ Levental, K. R., Yu, H., Kass, L., Lakins, J. N., Egeblad, M., Erler, J. T., Fong, S. F., Csiszar, K., Giaccia, A., Weninger, W., Yamauchi, M., Gasser, D. L., and Weaver, V. M. (2009) Matrix crosslinking forces tumor progression by enhancing integrin signaling. Cell 139, 891-906

7. Vogel, V. (2018) Unraveling the Mechanobiology of Extracellular Matrix. Annu Rev Physiol 80, 353-387

8. Silver, F. H., Freeman, J. W., and Seehra, G. P. (2003) Collagen self-assembly and the development of tendon mechanical properties. J Biomech 36, 1529-1553

9. Petruska, J. A., and Hodge, A. J. (1964) A Subunit Model for the Tropocollagen Macromolecule. Proc Natl Acad Sci U $S$ A 51, 871-876

10. Ryan, C. N. M., Sorushanova, A., Lomas, A. J., Mullen, A. M., Pandit, A., and Zeugolis, D. I. (2015) Glycosaminoglycans in Tendon Physiology, Pathophysiology, and Therapy. Bioconjugate Chemistry 26, 1237-1251

11. Gandhi, N. S., and Mancera, R. L. (2008) The structure of glycosaminoglycans and their interactions with proteins. Chem Biol Drug Des 72, 455-482

12. Orgel, J. P., Eid, A., Antipova, O., Bella, J., and Scott, J. E. (2009) Decorin core protein (decoron) shape complements collagen fibril surface structure and mediates its binding. PLoS One 4, e7028

13. Sweeney, S. M., Guy, C. A., Fields, G. B., and San Antonio, J. D. (1998) Defining the domains of type I collagen involved in heparin- binding and endothelial tube formation. Proc Natl Acad Sci U S A 95, 7275-7280

14. San Antonio, J. D., Karnovsky, M. J., Gay, S., Sanderson, R. D., and Lander, A. D. (1994) Interactions of syndecan-1 and heparin with human collagens. Glycobiology 4, 327-332

15. Scott, J. E., and Glanville, R. W. (1993) Homologous sequences in fibrillar collagens may be proteoglycan binding sites. Biochem Soc Trans 21, $123 \mathrm{~S}$

16. Sasaki, N., and Odajima, S. (1996) Elongation mechanism of collagen fibrils and force-strain relations of tendon at each level of structural hierarchy. J Biomech 29, 1131-1136

17. Rigozzi, S., Muller, R., Stemmer, A., and Snedeker, J. G. (2013) Tendon glycosaminoglycan proteoglycan sidechains promote collagen fibril sliding-AFM observations at the nanoscale. $J$ Biomech 46, 813-818

18. Magerle, R., Dehnert, M., Voigt, D., and Bernstein, A. (2020) Nanomechanical 3D Depth Profiling of Collagen Fibrils in Native Tendon. Analytical Chemistry 92, 8741-8749

19. Hackett, A. R., and Lee, J. K. (2016) Understanding the NG2 Glial Scar after Spinal Cord Injury. Frontiers in Neurology 7

20. Lewis, P. N., Pinali, C., Young, R. D., Meek, K. M., Quantock, A. J., and Knupp, C. (2010) Structural Interactions between Collagen and Proteoglycans Are Elucidated by Three-Dimensional Electron Tomography of Bovine Cornea. Structure 18, 239-245

21. Naylor, E. C., Watson, R. E. B., and Sherratt, M. J. (2011) Molecular aspects of skin ageing. 69, 249-256

22. Iozzo, R. V., and Gubbiotti, M. A. (2018) Extracellular matrix: The driving force of mammalian diseases. Matrix Biol 71-72, 1-9 
23. Murienne, B. J., Chen, M. L., Quigley, H. A., and Nguyen, T. D. (2016) The contribution of glycosaminoglycans to the mechanical behaviour of the posterior human sclera. JR Soc Interface 13, 20160367

24. Law, B., Fowlkes, V., Goldsmith, J. G., Carver, W., and Goldsmith, E. C. (2012) Diabetes-Induced Alterations in the Extracellular Matrix and Their Impact on Myocardial Function. Microscopy and Microanalysis 18, 22-34

25. Dufrene, Y. F., Ando, T., Garcia, R., Alsteens, D., Martinez-Martin, D., Engel, A., Gerber, C., and Muller, D. J. (2017) Imaging modes of atomic force microscopy for application in molecular and cell biology. Nat Nanotechnol 12, 295-307

26. Krieg, M., Fläschner, G., Alsteens, D., Gaub, B. M., Roos, W. H., Wuite, G. J. L., Gaub, H. E., Gerber, C., Dufrêne, Y. F., and Müller, D. J. (2019) Atomic force microscopy-based mechanobiology. Nature Reviews Physics 1, 41-57

27. Wenger, M. P., Bozec, L., Horton, M. A., and Mesquida, P. (2007) Mechanical properties of collagen fibrils. Biophys $J$ 93, $1255-1263$

28. Grant, C. A., Brockwell, D. J., Radford, S. E., and Thomson, N. H. (2009) Tuning the elastic modulus of hydrated collagen fibrils. Biophys J 97, 2985-2992

29. Peacock, C. J., and Kreplak, L. (2019) Nanomechanical mapping of single collagen fibrils under tension. Nanoscale 11, 14417-14425

30. Strasser, S., Zink, A., Janko, M., Heckl, W. M., and Thalhammer, S. (2007) Structural investigations on native collagen type I fibrils using AFM. Biochem Biophys Res Commun 354, 27-32

31. Uhlig, M. R., and Magerle, R. (2017) Unraveling capillary interaction and viscoelastic response in atomic force microscopy of hydrated collagen fibrils. Nanoscale 9, 1244-1256

32. Baldwin, S. J., Quigley, A. S., Clegg, C., and Kreplak, L. (2014) Nanomechanical mapping of hydrated rat tail tendon collagen I fibrils. Biophys J 107, 1794-1801

33. Coulson-Thomas, V. J., and ferreira, t. (2014) Dimethylmethylene Blue Assay (DMMB). Bio-protocol 4, e1236

34. Andriotis, O. G., Desissaire, S., and Thurner, P. J. (2018) Collagen Fibrils: Nature's Highly Tunable Nonlinear Springs. ACS Nano 12, 3671-3680

35. Karlsson, J. O., Andersson, M., Berntsson, P., Chihani, T., and Gatenholm, P. (1998) Swelling behavior of stimuliresponsive cellulose fibers. Polymer 39, 3589-3595

36. Scott, J. E., and Orford, C. R. (1981) Dermatan sulphate-rich proteoglycan associates with rat tail-tendon collagen at the $\mathrm{d}$ band in the gap region. 197, 213-216

37. Broom, N. D., and Poole, C. A. (1983) Articular cartilage collagen and proteoglycans. Their functional interdependency. Arthritis Rheum 26, 1111-1119

38. Skandalis, S. S., Theocharis, A. D., Vynios, D. H., Theocharis, D. A., and Papageorgakopoulou, N. (2004) Proteoglycans in human laryngeal cartilage. Identification of proteoglycan types in successive cartilage extracts with particular reference to aggregating proteoglycans. Biochimie 86, 221-229

39. Sader, J. E., Chon, J. W. M., and Mulvaney, P. (1999) Calibration of rectangular atomic force microscope cantilevers. Review of Scientific Instruments 70, 3967-3969

40. Necas, D., and Klapetek, P. (2012) Gwyddion: an open-source software for SPM data analysis. Cent Eur J Phys 10, 181-188

41. Andriotis, O. G., Manuyakorn, W., Zekonyte, J., Katsamenis, O. L., Fabri, S., Howarth, P. H., Davies, D. E., and Thurner, P. J. (2014) Nanomechanical assessment of human and murine collagen fibrils via atomic force microscopy cantilever-based nanoindentation. J Mech Behav Biomed Mater 39, 9-26

\section{Footnotes}

${ }^{1}$ The abbreviations used are:

ECM, extracellular matrix; GAG, glycosaminoglycan; PG, proteoglycan; AFM, atomic force microscopy; AM-AFM, amplitude-modulation AFM; FFM-AFM, fast force mapping AFM; DMMB, dimethylmethylene blue; InVOLS, inverse optical lever sensitivity; ANOVA, analysis of variance. 
Figures and Table.
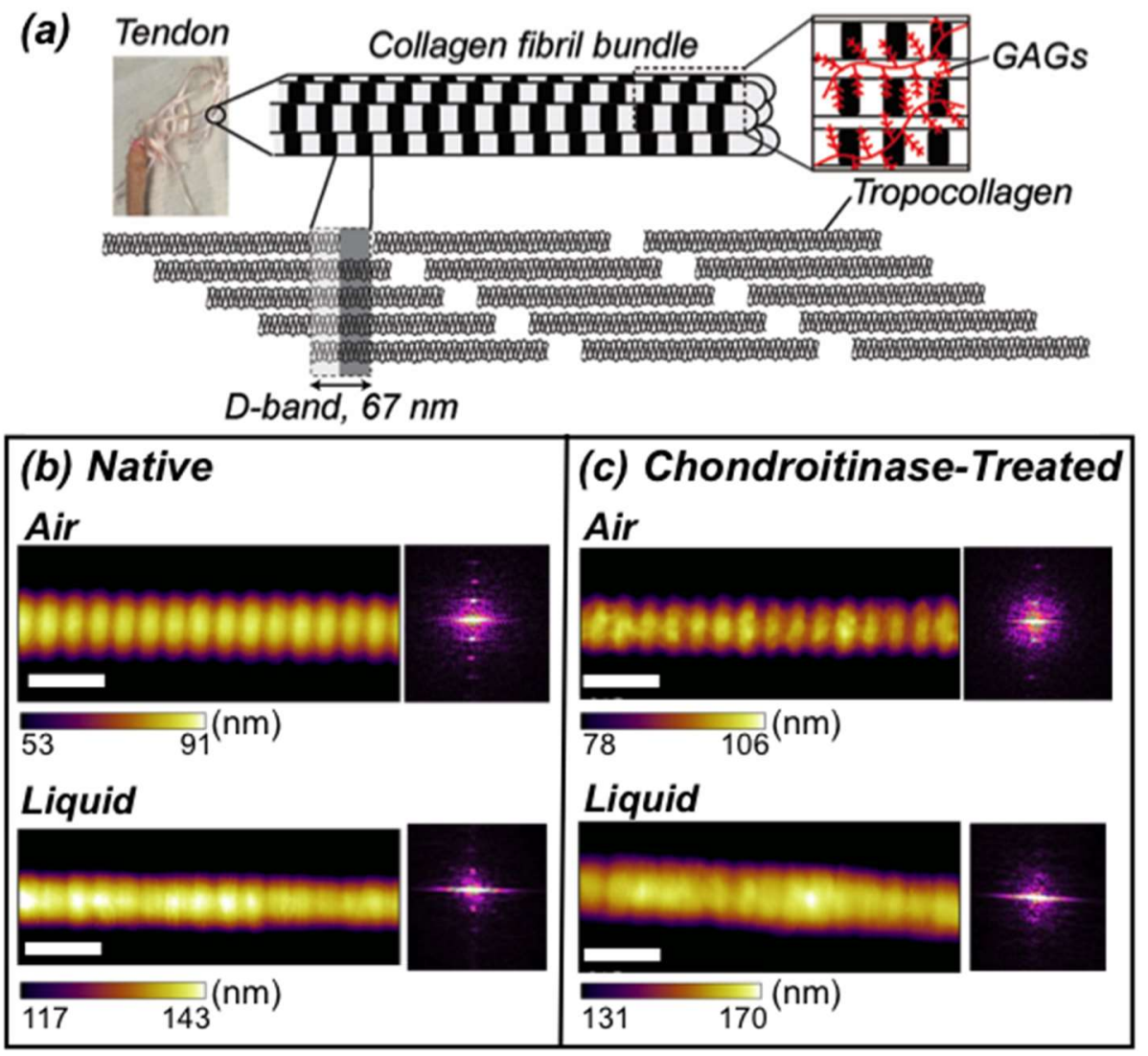

Figure 1. Collagen fibril D-banding. (a) Schematic of fibrillar collagen hierarchy from rat tail tendons to triple helices. The major protein component of tendon ECM is collagen $I$ in the form of fibril bundles, or fibers. PGs (red) displaying GAGs interact with individual fibrils within a fibril bundle. The characteristic D-bands are produced by the staggered arrangement of triple helical tropocollagens, resolved here by AM-AFM experiments for both (b) native and (c) chondroitinase-treated collagen fibrils imaged in air and liquid environments. The white scale bars are $200 \mathrm{~nm}$ in each image. The Fourier transform insets show the periodicity of each fibril and scale bar ranges were chosen to accentuate the D-banding in the images. Uncropped height, amplitude, and phase images are shown in the SI (Figure S2, S3). 
(a) Native

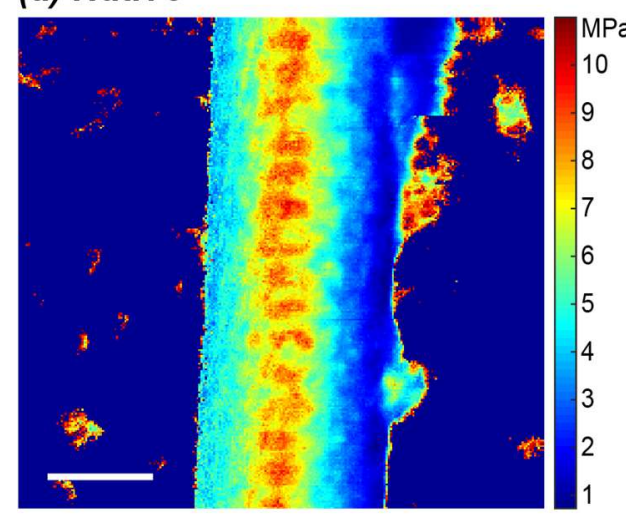

(b) Chondroitinase - Treated

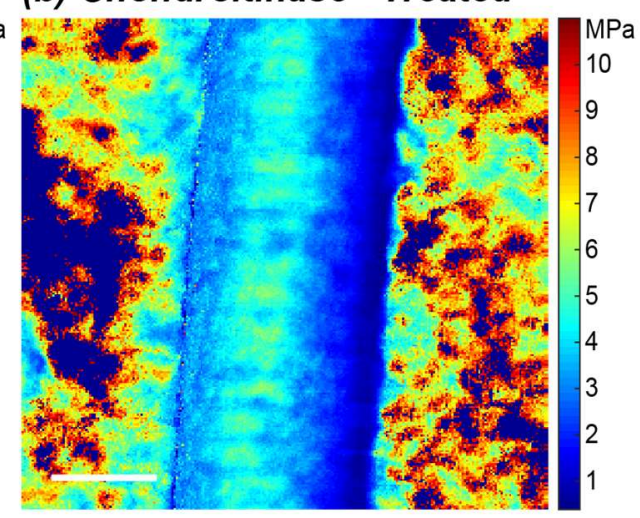

(c) Ind. Modulus

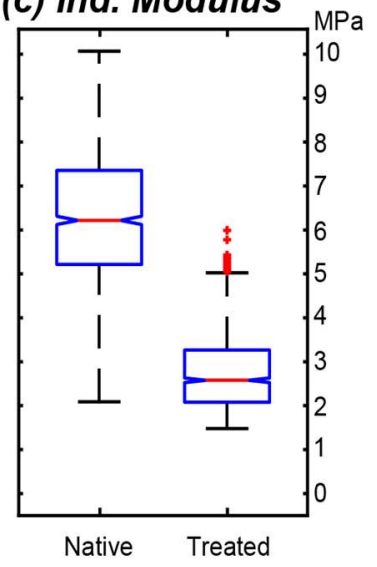

Figure 2. Nanoscale indentation modulus maps of collagen fibrils in solution. $(\mathrm{a}, \mathrm{b})$ Representative $1 \mathrm{x}$ $1 \mu \mathrm{m}$ fast force maps of individual (a) native, untreated and (b) chondroitinase-treated, GAG-depleted collagen fibrils from FFM-AFM experiments. The color at each pixel shows the local nanoscale indentation moduli ( 1 pixel / every $\sim 15 \mathrm{~nm}^{2}$ ); the FFM images are spatial reconstructions of 65,536 discrete force-distance curves that were fitted using standard indentation protocols (41) and subsequently replotted in Cartesian space using in-house MATLAB code. A large scan size was selected to capture more than ten D-bands per image, enabling meaningful comparisons between internal regions, fibrils, and treatments. White scale bars indicate $200 \mathrm{~nm}$. (c) Box and whisker plots compare a subset of indentation moduli data in (a) and (b) for force curves acquired only at the apex down the fibril long axes. In (c), data for five fibrils in each condition are presented for increased statistical confidence. 
Table 1: Overview of results for the control and chondroitinase treated fibrils. Visualization of the distribution of these values are shown in the SI (Figure S7).

\begin{tabular}{|l|c|c|}
\cline { 2 - 3 } \multicolumn{1}{c|}{} & Control & Treated \\
\hline Periodicity (air) & $65.6 \pm 1.0 \mathrm{~nm}$ & $66.0 \pm 0.2 \mathrm{~nm}$ \\
\hline Periodicity (liquid) & $65.8 \pm 0.8 \mathrm{~nm}$ & $66.2 \pm 1.0 \mathrm{~nm}$ \\
\hline $\begin{array}{l}\text { Overlap-Gap } \\
\text { Height Difference (air) }\end{array}$ & $3.6 \pm 2.4 \mathrm{~nm}$ & $3.6 \pm 2.7 \mathrm{~nm}$ \\
\hline Hydration Ratio & $1.79 \pm 0.06$ & $1.9 \pm 0.2$ \\
\hline Modulus (liquid) & $6.4 \pm 1.4 \mathrm{MPa}$ & $2.9 \pm 1.0 \mathrm{MPa}$ \\
\hline
\end{tabular}

